\title{
Digitalisierung und künstliche Intelligenz - wie behalten wir das ethisch im Griff?
}

\author{
Ethik und künstliche Intelligenz, wie passt das zusammen? \\ Im Artikel erfahren Sie, welche Überlegungen aus der Wissenschaft \\ der Ethik notwendig sind, um Digitalisierung, Automatisierung \\ und Roboterisierung so zu gestalten und zu entwickeln, dass eine \\ menschen- und naturgerechte Digitalisierung möglich ist. \\ Von Ulf Posé
}

r ede technische Neuerung bietet enorme Chancen und Risiken. Gleichgültig, welche Entwicklung die Menschen nach vorn gebracht hat. Es kam immer zu Missbräuchen und Fehlern, die teuer waren und auch viele Menschenleben gekostet haben. Daran wird auch die künstliche Intelligenz nichts ändern.

Kritische Stimmen müssen erlaubt sein. Eine Warnung kann auf Gefahren aufmerksam machen. Jedoch sind Warnungen und kritische Stimmen nicht die Lösung möglicher Probleme durch die künstliche Intelligenz.

Aus ethischer Sicht werden wir uns weltweit fragen müssen, welche ethischen Standards lassen sich in Algorithmen erfassen und einbauen? Welche Handlungs- und Entscheidungskontrollen müssen Menschen behalten, um in einer Güterabwägung zu ethisch verantworteten Entwicklungen der künstlichen Intelligenz beizutragen. Letztlich geht es, ethisch betrachtet, bei der künstlichen Intelligenz nur um zwei Dinge:

- Mit welchen Konsequenzen ist zu rechnen, die sich für Menschen, für die Umwelt ergeben, wenn wir mit den sogenannten ,intelligenten“ Automaten, Robotern, Computern umgehen?

- Welche Verantwortlichkeiten haben wir, wenn wir Roboter, Computer, Automaten einsetzen?

Die Angst vor künstlicher Intelligenz, Roboterisierung und Automatisierung sollte uns nicht davon abhalten, schon beim Schreiben von Algorithmen, beim Programmieren ethische Standards zu berücksichtigen. Denn eines ist sicher: Ein Roboter, ein Automat, die gesamte künstliche Intelligenz tut nur das, was der Mensch programmiert und möglich gemacht hat. Insofern liegt die Verantwortung immer beim Menschen. Ein Roboter, ein Algorithmus ist das Ergebnis menschlicher Kreativität. Selbst wenn Computer heute schon selbstständig Algorithmen schreiben, sie tun es nur, weil Menschen es möglich gemacht haben, dazu passende Programme entwickelt haben.

\section{Ethische Standards}

Eines bleibt ebenfalls wichtig. Computer können noch so schnell agieren, der menschlichen Intelligenz sind sie nicht in allen Facetten überlegen. Was dem Computer gegeben ist, ist eine klare Kosten-Nutzen-Analyse und -Kalkulation. Menschliche Intelligenz ist mehr. Freude empfinden, Ärger oder Begeisterung, das lässt sich (derzeit) nicht so programmieren, dass es mit dem menschlichen Empfinden identisch ist. Menschen können ihre Gefühle empfinden, und über sie nachdenken. Der Geistesblitz, über dessen Entstehen es keine Untersuchung gibt, ist dem Computer nicht möglich. Er könnte es nur, wenn er darauf programmiert wäre. Dann ist es aber kein Geistesblitz mehr. Menschen können über sich nachdenken, ohne da- rauf programmiert worden zu sein. Und sie können über das Nachdenken nachdenken. Das Bewusstsein „Ich bin ein Computer", fehlt dem Computer. Das muss ihm einprogrammiert werden. Ein Computer mag nach unseren Vorstellungen über so etwas wie Verstand verfügen, über Sinnlichkeit verfügt er nicht. Die große Frage ist, ob sich solch ein Bewusstsein über Algorithmen überhaupt programmieren lässt.

\section{Programmierung und Tugend}

Hier kommt Ethik ins Spiel. Ethik will sicherstellen, dass das menschliche Zusammenleben anhand von Normen, Regeln und Standards zur Lebensmehrung führt. Ethik will immer eine sittliche Verbesserung des konkreten Menschen erreichen. Dazu gilt es, den Verstand, den Charakter, die Tugenden und Emotionen eines Menschen zu betrachten. Dass ein Computer Charakter besitzt, davon habe ich bis heute nichts gehört. Und auch von Tugenden eines Computers ist mir nichts bekannt.

Informatik, als Wissenschaft von der systematischen Verarbeitung von Informationen, besonders der automatischen Verarbeitung mithilfe von Digitalrechnern, schaute lange nicht auf den Menschen. Informatik sorgt letztlich für eine systematische Verarbeitung von Informationen. Immer mit der Aufgabe, Informationen in eine Rechenanlage $\mathrm{zu}$ implementieren, um diese aufzubauen oder weiterzuentwickeln. Nicht wenige Wissenschaftler/innen sind sich heute einig und überzeugt davon, dass Mensch und Maschine eine Beziehung eingehen und diese Beziehung auch betrachtet und gestaltet werden muss. Schon Nyrgaard meinte: "To program is to understand! "Heute wissen wir um die Auswirkungen von Maschinen auf Menschen. Wir können zunehmend wahrnehmen, welche Auswirkungen und Bedeutungen Computer, Roboter, Automaten auf Menschen, auf das soziale Miteinander, auf eine Gesellschaft, auf die Umwelt haben. Damit kann Informatik als Wissenschaft nicht mehr isoliert betrachtet werden. 
So bleibt die wichtige Aufgabe, wie muss Programmierung sein, damit sie ethischen Standards genügt. SAP zum Beispiel (und viele andere mehr) hat einen Verhaltenskodex entworfen, der ethische Standards im Umgang mit künstlicher Intelligenz beinhaltet. Das ist die Zukunft der IT-Spezialist/innen. Neben allem technischen Know-how auch ethisches Know-how in Algorithmen zu verarbeiten. Die IT-Industrie, die Wissenschaft und die Politik denken hoffentlich noch intensiver darüber nach.

Dazu wird es notwendig sein:

- Informatik neu zu definieren als eine Wissenschaft, die nicht nur wissenschaftstheoretische Bedeutung, sondern auch gesellschaftliche und philosophische Bedeutung hat. Bisher war der Mensch in seinem Menschsein Gegenstand der Philosophie. Es wird erforderlich sein, Menschsein noch mehr zum Gegenstand der Informatik werden zu lassen.

- ethische Standards zu definieren. Eine Arbeit, die der UN in der Umsetzung derzeit noch schwerfällt, auch wenn wir definierte Standards im code of conduct oder der UN-Menschenrechtscharta haben.

- ethische Standards in Konkurrenz zu ökonomischen, ökologischen, sozialen Standards in definierten Güterabwägungen abzubilden.

- ethische Standards zu entwickeln, die Diskriminierungen verhindern. Schon heute sind Programme in der Lage, den Informationsfluss zu Personen oder Gesellschaften zu reglementieren.

- Projekte zu definieren, in denen IT-Spezialisten mit Ethikexperten gemeinsam ein ethisches Profil für Algorithmen, Automaten, Roboter entwickeln.

- Dilemmata-Lösungen für künstliche Intelligenz $\mathrm{zu}$ entwickeln, die technisch machbar und ethisch vertretbar sind.

- den Pluralismus zu erhalten. Die Digitalisierung sorgt dafür, dass Informationen immer gezielter und individueller an Nutzer/innen herangetragen werden. Damit fällt die Meinungsbil- dung immer schwerer. Es gilt, ethische Standards zu entwickeln, die fundierte Meinungsbildung auch möglich machen.

I sicherzustellen, dass Menschen Verantwortung nicht an Maschinen delegieren können. Damit ist es notwendig, die absolute Autonomie von Robotern, Computern und Automaten zu verhindern.

】 den „gläsernen Menschen“ zu verhindern. Datenschutzgesetze müssen sicherstellen, dass die Datenmenge über einen Menschen vom Einzelnen selbst bestimmbar bleibt.

- die Umkehrbarkeit von Entscheidungen/Programmierungen sicherzustellen.

\section{Vorteile von künstlicher Intelligenz}

Die Vorteile, die künstliche Intelligenz uns bieten wird und kann, sind gigantisch und heute noch nicht absehbar. Die möglichen Gefahren sind auch zum großen Teil Spekulation; gleichwohl gilt es, das zu identifizieren, was derzeit zu identifizieren ist, und vorsorglich damit umzugehen. Seit Anfang 2017 gibt es bereits einen Resolutionsentwurf des EU-Parlaments, der sich mit der zivilrechtlichen Regelung im Bereich Robotik und künstliche Intelligenz befasst hat. Das EU-Parlament befasst sich hier mit der Vorsorge. In der Empfehlung heißt es: „dass die künstliche Intelligenz die intellektuellen Fähigkeiten des Menschen [...] in einer Weise überflügeln könnte, die [...] die Fähigkeit der Menschheit, ihre eigene Schöpfung zu kontrollieren und folglich vielleicht auch die Fähigkeit, Herr über ihr eigenes Schicksal zu bleiben und für das Überleben der Spezies Mensch Sorge tragen zu können, ernsthaft gefährden könnte.“ Diese ernsthafte Gefährdung sieht einen kill switch für Roboter vor. Kill switch meint, es muss immer die Möglichkeit geben, einen Roboter oder Computer auch abstellen zu können.

Auch der Physiker Stephen Hawkings hat gewarnt und gleichzeitig den Informatiker/innen eine Handlungsanwei- sung gegeben: „Der Erfolg bei der Schaffung einer effektiven künstlichen Intelligenz könnte das größte Ereignis in der Geschichte unserer Zivilisation sein. Oder das Schlimmste. Wir wissen es einfach nicht. Also können wir nicht wissen, ob wir unendlich von der künstlichen Intelligenz unterstützt oder ignoriert, gefüttert oder möglicherweise zerstört werden. Wenn wir nicht lernen, uns auf mögliche Gefahren vorzubereiten und sie zu vermeiden, könnte künstliche Intelligenz das schlimmste Ereignis in der Geschichte unserer Zivilisation sein. Sie bringt Gefahren mit sich, wie mächtige autonome Waffen oder neue Wege für die Wenigen, die Vielen zu unterdrücken.“

Noch eine Aufgabe haben wir. Es wird sehr darauf ankommen, digital fit zu sein. Wir werden dafür Sorge tragen müssen, die Menschen in die Lage zu versetzen, mit Computern umzugehen. Es geht um die Urteilsfähigkeit. Wir werden aufhören müssen, Ahnungen und Befürchtungen $\mathrm{zu}$ erzeugen und zu schüren. Es geht darum, Wissen und Können im Umgang mit Computern so zu vermitteln, dass der User urteilsfähig bleibt.

Es wird also darauf ankommen, wie die künstliche Intelligenz werden wird, welche ethischen Standards programmiert werden und wie wir sicherstellen, dass diese ethischen Standards auch überprüft werden. Dazu ist es erforderlich, die möglichen Gefahren zu ermitteln und deren Abwehr soweit wie möglich zu organisieren. Das wird nicht einfach sein. Aber vielleicht können uns Computer dabei helfen. Geben wir ihnen eine Chance.

AUTOR + KONTAKT

Ulf Posé ist Managementtrainer, Coach und Keynote-Speaker. Er ist Vorsitzender des Ethikbeirats im Senat der Wirtschaft und Autor von 24 Büchern zur Unternehmens- und Lebensführung. Posétraining, Klosterstr. 19-21, 41189 Mönchengladbach. Tel.: +49 2166 57841, E-Mail: infor@posetraining.de, Website: www.posetraining.de 